LAWRENCE LIVERMORE N A T IO N A L LABORATORY
Measurement of Shear Strength in BCC Materials Subjected to Moderate Pressures

J.P. Escobedo, D.P. Field, D.H. Lassila, M.M. Leblanc, B.P. Bonner

February 24, 2004 
This document was prepared as an account of work sponsored by an agency of the United States Government. Neither the United States Government nor the University of California nor any of their employees, makes any warranty, express or implied, or assumes any legal liability or responsibility for the accuracy, completeness, or usefulness of any information, apparatus, product, or process disclosed, or represents that its use would not infringe privately owned rights. Reference herein to any specific commercial product, process, or service by trade name, trademark, manufacturer, or otherwise, does not necessarily constitute or imply its endorsement, recommendation, or favoring by the United States Government or the University of California. The views and opinions of authors expressed herein do not necessarily state or reflect those of the United States Government or the University of California, and shall not be used for advertising or product endorsement purposes.

This work was performed under the auspices of the U.S. Department of Energy by University of California, Lawrence Livermore National Laboratory under Contract W-7405-Eng-48. 


\title{
Measurement of Shear Strength in BCC materials Subjected to Moderate Pressures
}

\author{
J.P. Escobedo ${ }^{1}$, D.P. Field ${ }^{1}$, D.H. Lassila ${ }^{2}$, M.M. Leblanc ${ }^{2}$, and B.P. Bonner ${ }^{2}$ \\ ${ }^{1}$ School of Mechanical and Materials Engineering, Washington State University, Pullman, WA. \\ ${ }^{2}$ Lawrence Livermore National Laboratory, Livermore, CA.
}

\begin{abstract}
An experimental procedure is reported to perform shear tests on specimens held under moderately high hydrostatic pressures (on the order of $10 \mathrm{GPa}$ ). The mechanical behavior of materials subjected to such pressures, varies substantially from that observed at atmospheric pressure or even pressures typically attained during industrial processing. These differences must be incorporated into models such as the Steinberg-Guinan hardening model or discrete dislocation dynamics simulations. The goal of the proposed research is to develop and implement testing procedures that experimentally determine pressure-dependent dislocation mobilities in oriented single crystals of the BCC transition metals. These experiments will provide calibration data for models of materials subjected to extreme pressures and will assist in model validation. This paper reports the development of the experimental procedures. A thin foil of polycrystalline Ta was used to perform the initial experiments under hydrostatic pressures ranging from 2.1 to $4.2 \mathrm{GPa}$. Both yielding and hardening behavior are observed to be sensitive to the imposed pressure.
\end{abstract}

Keywords: High pressure, Experimental mechanics, polycrystalline Ta.

\section{Introduction}

The study of material strength under ultrahigh pressures is an important subject due to the fact that almost all the knowledge we have of the materials comes from tests carried out at ambient pressure [1]. Of particular interest in the present work is material behavior at moderate pressures such as those attained during weak shock loading. Properties such as hardening and ductility of metals are sensitive to high pressures, even at relatively low pressures 0.7-3.0 GPa a remarkable increase in ductility of some materials has been reported [2], such is the case with tungsten, which is brittle at atmospheric pressure but can achieve elongations as high as $100 \%$ when subjected to a pressure of $2.8 \mathrm{GPa}$. Another property of interest is the shock-induced phase transformation. Numerous cases of phase changes have been reported when materials are subjected to pressures exceeding $30 \mathrm{GPa}[3,4]$.

Most of the high-pressure research is done under static conditions. For pressures in the range of $0-3 \mathrm{GPa}$ testing has been conducted using a variety of media including solid, liquids and gases [16]. Higher pressures are required to observe various material behaviors such as pressure-hardening which requires pressures on the order of at least 10 $\mathrm{GPa}$ according to Weir, et al [3].

To achieve higher pressures, experiments have been conducted using the diamond anvil cell, (Figure 1) [3, 4, 5], where the specimen is loaded to high pressures between the diamond anvils. Although this device allows ultrahigh pressures to be reached readily, it has the deficiency that the hydrostatic, frictional and deviatoric stresses increase in an uncontrolled manner as the load increases [6]. 


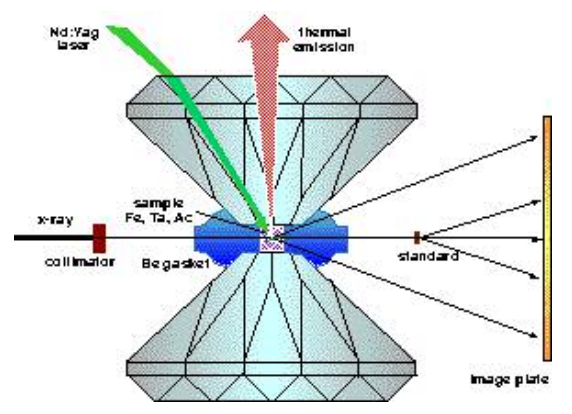

Figure 1 - Schematic of the diamond anvil cell (DAC), the sample is pressed between the anvils and an $x$ ray source is used to study the materials during the process.

Typically the volume of material tested in these kinds of systems is small and properties that are observed are sometimes functions of the sample size. Postmortem analysis in these types of experiments is often difficult or impossible because of the sample size. One goal of our experiment is to perform high pressure experiments using a larger specimen size that can be analyzed using standard characterization tools subsequent to testing.

For the experiments designed in the current research, two major features were desired; strict control in the loading path, in order to separate the effects of hydrostatic and deviatoric stresses, and the ability to perform post-mortem characterization such as hardness measurements and TEM analysis. To accomplish these goals a modified Bridgman cell was developed.

Of the various types of anvils developed by Bridgman for high pressure work, the one developed for applying a shearing load on specimens under high pressure most closely approximates the desired test objectives. This cell is described schematically in Figure 2.

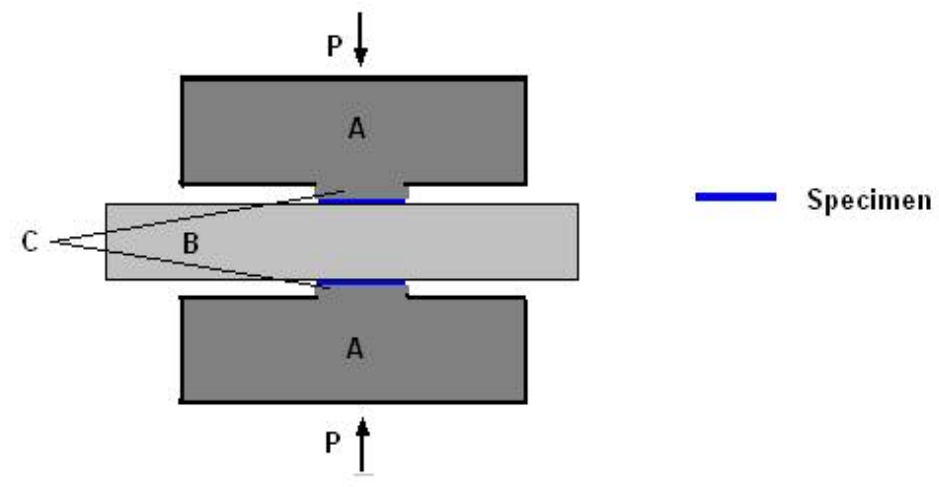

Figure 2 - Schematic of the original Bridgman anvil cell.

In Bridgman's original design [7], blocks A are two hardened steel cylindrical blocks bearing short bosses $\mathbf{C}$. B is a rectangular block of hardened steel. The specimens to be tested are small thin disks placed between block $\mathbf{B}$ and the bosses $\mathbf{C}$. Load is applied to blocks $\mathbf{A}$, causing the material to extrude laterally until an equilibrium thickness is 
reached. The block $\mathbf{B}$ then is rotated about the axis though the bosses $\mathbf{C}$. The experiment consists of measuring how much force is required to rotate as function of pressure.

\section{Experimental Development}

The experiment described in this work enables monitoring of the mechanical response of materials deformed in shear under hydrostatic pressure in the range of $0-50 \mathrm{GPa}$ depending upon the size of the specimens. Observation of the pressure-induced work hardening and, of special interest, measurement of the pressure-dependent dislocation mobility in oriented single crystals are goals of the experiment.

Using the concept of Bridgman's cell, a new high-pressure testing apparatus was designed and constructed. A schematic of the complete device is depicted in Figure 3. It consists basically of a set of upper platens attached to the crosshead of a biaxial materials testing system with the bottom platens free to perform vertical and angular displacements. Angular displacement is accomplished using a driving clevis attached to bottom part of the apparatus. A stationary sleeve rigidly attached to the upper platen and fitting with close tolerance over the lower platen ensures proper alignment between the mating surfaces while the load is applied. Brass rings were attached at the top and bottom of the lower barrel to avoid steel on steel friction during loading. To ensure that frictional forces would be negligible, a low viscosity lubricant, 30 weight oil, was spread on the brass rings.

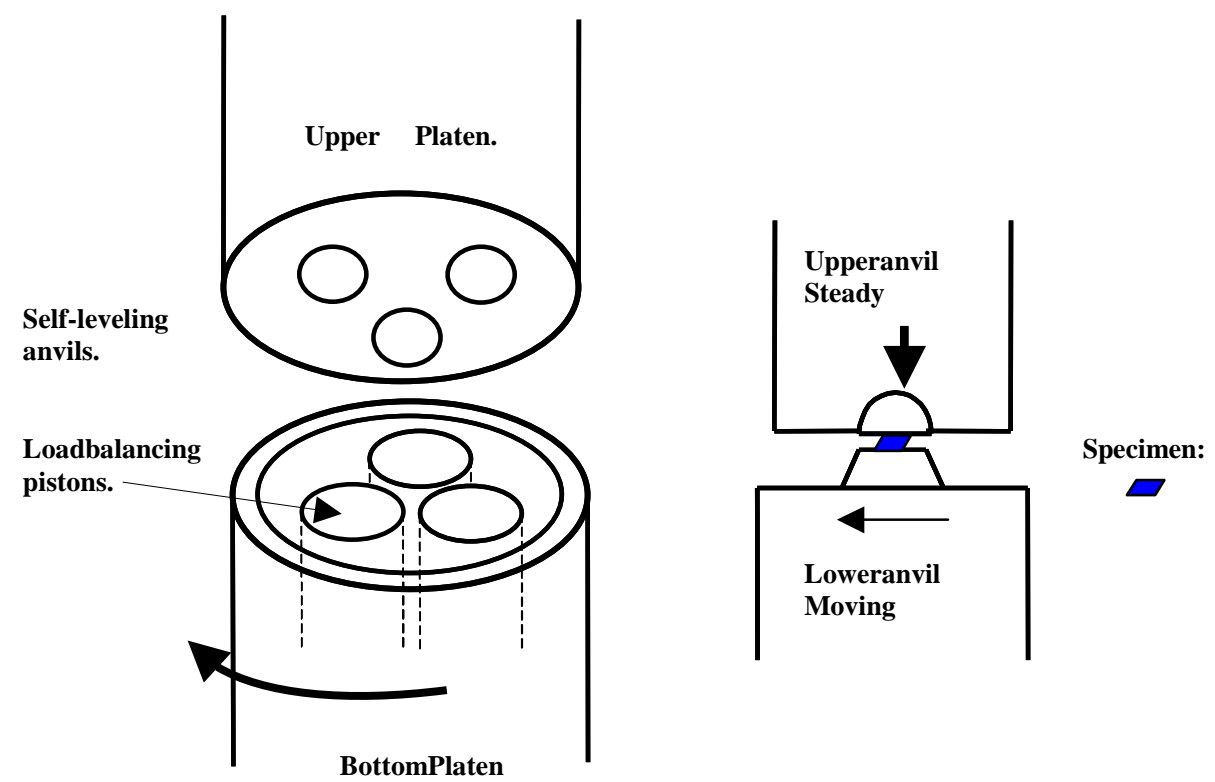

Figure 3 - Schematic of the modified Bridgman cell used to perform the experiments, the specimens are plastically deformed in shear by the torsion motion of the bottom platen. 
In this modified Bridgman cell, three independent supported anvils in both the top and bottom platens are arranged symmetrically. The anvil centers are all positioned on a circle centered on the loading axis of the testing system. The anvils in the top platen are formed with a hemispherical section that fits snugly into the mating surface. Using a thin foil of indium between these hemispherical surfaces provides for self-leveling of the anvils upon initial loading. The same pressure is attained between all anvils by positioning each anvil atop a hydraulically-controlled piston, all of which are connected to a common oil reservoir. For the "tri-anvil" testing apparatus the anvil material chosen was tungsten carbide.

The surfaces of the anvils were roughened (using 1200 grit emery cloth) to have better frictional contact with the specimens. This enables one to measure the strain of the specimens by measuring the displacement of the platens relative to each other. The surface roughness of the anvils was measured using atomic force microscopy (AFM) and a value of $750 \AA \mathrm{rms}$ was obtained. This value remains similar along the entire anvil surfaces, without noticeable changes between the area exposed to the specimen and the edges that are not in contact with the specimens during the experiments.

The deformation of the specimens in the tri-anvil apparatus is achieved using a four-step process:

First, the specimens are centered on each anvil using a sample positioning tool. The tool is specially designed for each specimen geometry and aligns the center of each specimen to within $25 \mu \mathrm{m}$ of the anvil center. The bottom platen is vertically displaced leaving a small gap between the specimens and the upper anvils.

Second, axial load is applied using load control and a rate of $2.2 \mathrm{kN} / \mathrm{sec}(500 \mathrm{lbf} / \mathrm{sec})$ once the desired load is reached, it is held constant. Although some slip in the surface of the material could be present at low pressures, when the pressure is high enough the surfaces of the specimen are welded to the roughened anvils (evidence of this is presented later in the discussion).

Once the specimens are under the desired pressure, the third step consists of rotational displacement applied to the bottom platen, causing essentially unidirectional shearing strains on the specimens positioned within each anvil, as shown in Figure 3. The shear loading can be assumed unidirectional because of the small specimen size and low angle rotations in comparison to the circumference of the circle on which the specimens lie. Extensometers were attached to the anvils to measure the local strain on the sample being deformed. This local measurement overcomes the complexity in strain measurement caused by concerns of machine compliance.

Finally, the specimens are unloaded and recovered for post-mortem analysis.

For converting the longitudinal displacement read by the extensometers to angular displacement imposed by the system, a linear fit between the extensometer displacement and a given rotation was obtained; a CAD program was used to simplify the process. 


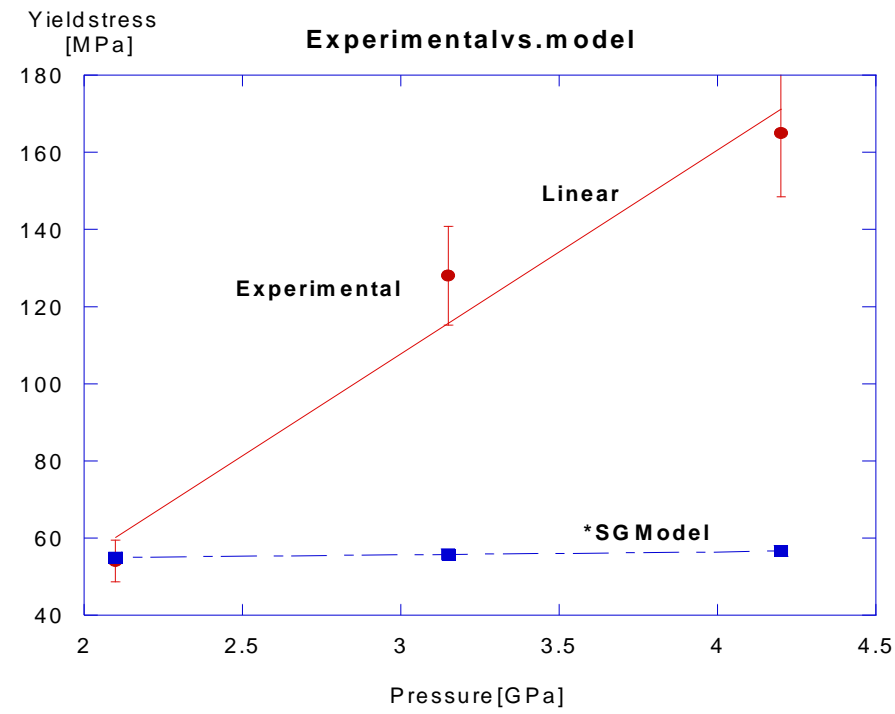

Figure 4 - Conversion from extensometer longitudinal to angle displacement

The graph shows the eight positions estimated and the linear fit used from here on to do the analysis for the preliminary tests. From this calculation a maximum angular displacement read by the ram of 0.6 degrees was calculated and used as the limit of deformation.

To verify that the torque measurements read by the system were due to the specimen tested and not intrinsic friction of the apparatus, a test without load and a rotation rate of 0.3 degrees / minute was performed. A value of $2.25 \pm 1.3$ in-lbf was read by the ram of the MTS machine.

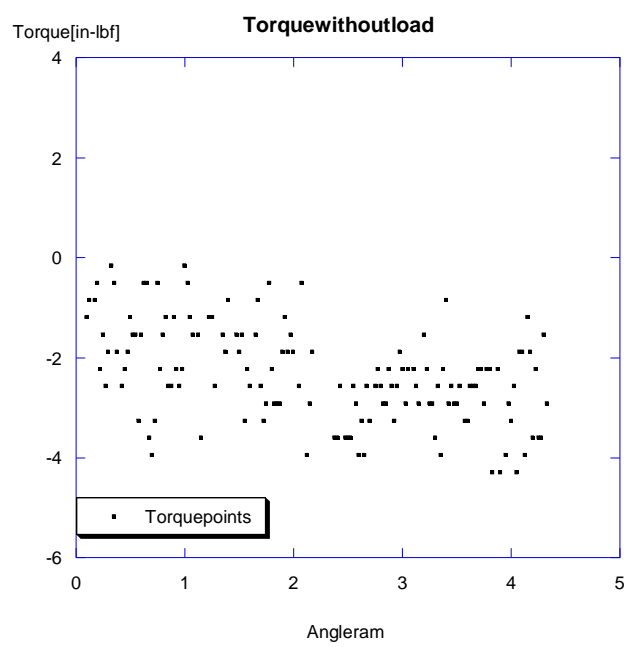

Figure 5 - Intrinsic torque of the testing apparatus.

The expected shear stress for the specimens is expected to be at least $50 \mathrm{MPa}$ [4], translated to a torque of $400 \mathrm{in}-\mathrm{lbf}$. Therefore, the intrinsic torque is on the order of $1 \%$, and can be neglected.

\section{Validation of the Experiment}


Plastic deformation in metallic systems occurs primarily via dislocation generation and movement due to shear stresses. The effective stress $\bar{\sigma}$ is equal to $\sigma_{y}$, the yield strength in tension. The effective stress is given as

$$
\bar{\sigma}=\frac{1}{\sqrt{2}}\left[\left(\sigma_{1}-\sigma_{2}\right)^{2}+\left(\sigma_{2}-\sigma_{3}\right)^{2}+\left(\sigma_{3}-\sigma_{1}\right)^{2}\right]^{1 / 2}
$$

where $\sigma_{1}, \sigma_{2}$, and $\sigma_{3}$ represent the principal stresses. In the case of hydrostatic pressure, $\sigma_{1}=\sigma_{2}=\sigma_{3}$, which results in no shear stresses present to provide a driving force for dislocation motion, therefore structural properties are not changed due to hydrostatic pressure alone.

In order to determine whether the desired state of hydrostatic pressure could be attained by applying axial load on the thin foil specimens, a finite element simulation was run using hydrodynamic lubrication theory applied to metals. The foils used in the experiment have a diameter of $3 \mathrm{~mm}$ and a thickness of 50 microns, so this geometry was used in the simulation. The result from the FE simulation showed no deformation in the major part of the specimen, with end effects limited to the very near end regions. This result is consistent with the fact that the decay length for the free surface effect, Dx, is considerably larger than the specimen diameter. The decay length is given by $\Delta x=\frac{h}{2} \frac{p}{\tau_{\operatorname{Max}}}$,

where $p$ is the axial pressure on the order of GPa, $h$ is the height of the foil and $\tau_{\text {Max }}$ is the maximum shear stress. This analysis offers assurance that the pressure is equally distributed along the entire specimen, with only minor end effects.

To experimentally verify the accuracy and adequacy of the trianvil experiment to perform measurements of dislocation mobility on oriented single crystals, initial tests were performed on polycrystalline tantalum samples over pressures of 2.1, 3.15 and 4.2 GPa. The specimens to be tested were obtained from the foil using a punch designed for TEM sample preparation. The geometry of the specimens was disk-shaped with a diameter of $3.0 \mathrm{~mm}$ and a nominal thickness of $50 \mu \mathrm{m}$.

Automated electron backscatter diffraction (EBSD) was used to characterize the initial structure of the annealed Ta foils. This technique reveals the texture and grain size distribution of the material as well as spatially specific information such as structural gradients and misorientation distributions.

As seen in Figure 6a, the initial microstructure is mainly a combination of two texture components, (111) and (001) as expected for rolled and annealed BCC metal. Figure 6b contains the grain size distribution that shows an average grain diameter of about $20 \mu \mathrm{m}$. 

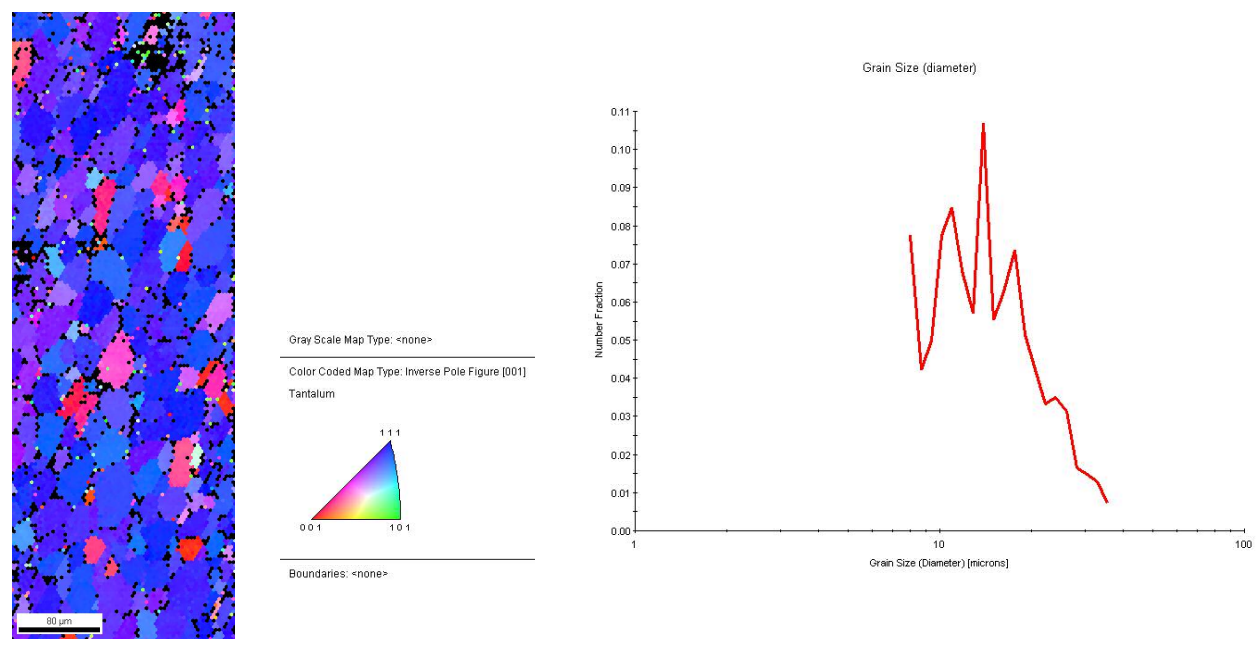

Figure 6 a-Orientation map of the as-received Ta foil, $b$-grain size distribution.

Specimens of this material were subjected to two different types of tests. The first imposed only a hydrostatic pressure, with no shearing imposed. These specimens were used as controls to determine the amount and distribution of plastic deformation suffered by the specimens during imposition of the hydrostatic pressure alone. The second set of tests involved shearing the specimens to strains on the order of 2-6 while subjected to a given hydrostatic pressure. For the sheared specimens an angular rate of 0.3 degrees $/ \mathrm{min}$ was imposed using the ram rotation as the control signal.

It was anticipated that when pressure is applied, the material deforms elastically until an equilibrium thickness, determined by the elastic properties of the material, is reached [12], , and hydrostatic pressure is distributed inside the specimen. To determine whether this objective was accomplished by the given specimen geometry and experimental approach, various analyses were performed. First, if the material is only deformed elastically during hydrostatic loading, the shape change of the loaded and unloaded specimens will be minimal (except near the edges). In addition, dislocation activity causes strain hardening in the material, so if significant dislocation activity occurs the hardness of the metal after loading could be expected to increase. Finally, direct observation of the microstructures by TEM or other means should reveal the microstructural changes due to dislocation activity. The absence of dislocation activity will ensure that the test objectives were achieved.

It was observed that the specimens in all cases were lens shaped after the load was imposed and released, being thinner at the edges than at the center. This is due to material extrusion near the edges to equilibrate the normal load and does not adversely affect the test if a state of hydrostatic pressure is attained over a significant portion of the specimen centers. Optical microscopy was used to characterize the thickness change for specimens loaded in hydrostatic pressure alone, and those deformed to high shearing strains. Figure 7 contains optical micrographs of the polished foil cross sections for the original foils (a), the foils loaded to $4.2 \mathrm{GPa}$ without shearing (b and c), and the specimens loaded to 4.2 GPa plus a shear strain of about 4 ( $\mathrm{d}$ and e). 

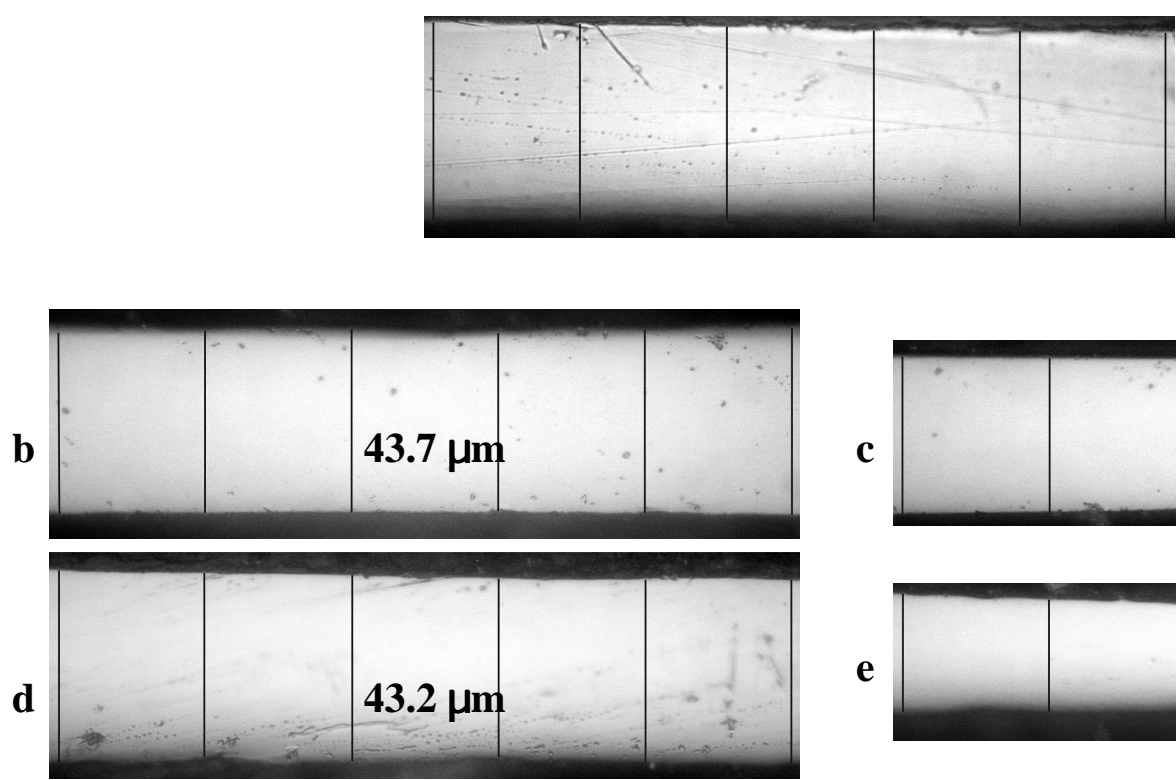

c

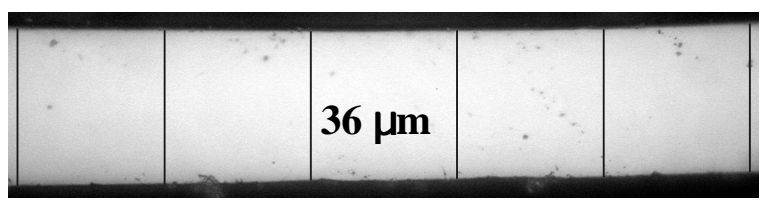

e

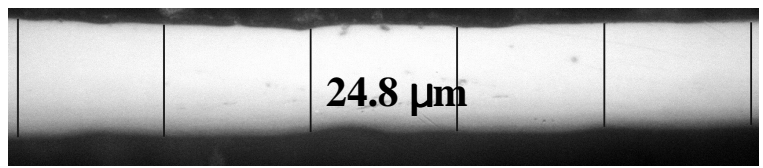

Figure 7 - Optical micrographs of the polished foil cross sections for the original foils (a), the foils loaded to 4.2 GPa without shearing (b-center and c-near edge), and the specimens loaded to 4.2 GPa plus a shear strain of about 4 (d-center and e-near edge).

The original foils had a nominal thickness of $50 \mu \mathrm{m} \pm 10 \%$ as specified by the manufacturer. The values measured via optical microscopy yielded a mean thickness of approximately $47 \mu \mathrm{m}$ for the original specimens.

For the specimens loaded only with hydrostatic pressure, no shear bands are observed and a decrease in the cross section is appreciated in the two localizations, being of about $8 \%$ reduction in the cross section for the center of the specimen, and getting almost $20 \%$ at the edge. For the sheared specimens, the center of cross section remained almost constant with a reduction close to $10 \%$, slightly different from the axially loaded, but some shear bands can be lightly appreciated. For the edge of the sheared specimens the thickness reduces dramatically being almost $48 \%$ of the original, and also some shear bands can be seen. Regarding the effect of the hydrostatic pressure, the optical micrographs (Fig 7.b and c) show that under hydrostatic pressure the center of the specimens remain almost not-deformed, but with some extrusion occurring at the edges of the sample.

Measurement of microhardness profiles across the specimen diameter is another way that dislocation activity during hydrostatic loading can be analyzed. A Vickers hardness apparatus was used to measure the point to point variation in mechanical properties of the samples subjected to pressures of 2.1 and $4.2 \mathrm{GPa}$. The load used in taking the hardness measurements was 100 grams. 


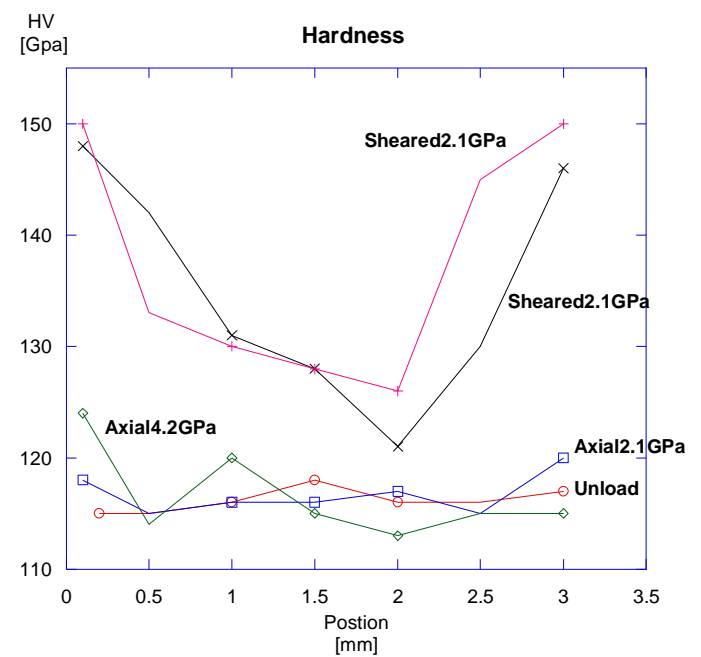

Figure 8 - Graph showing the hardening effect due to the pressure applied.

The microhardness measurements were done using steel holders as the substrates for the deformed specimens. The specimens were glued to the holders and pressure was applied in order to flatten the surface making it suitable for analysis. Hardness measurements were made every $0.5 \mathrm{~mm}$ from side-to-side acrossthe specimen .

For the original, well-annealed, foils the average hardness measured was $116 \mathrm{GPa}$. In the major part of the specimens that were subjected only to load, a measurement of the hardness was read close to the original material with a slight increase being noted at the ends of the specimens. Such an increase might be expected due to material extrusion near the edges where unconstrained flow of the material could occur.

For the specimens deformed to a shear strain close to 4 while maintained at pressure, a substantial increase in hardness is noted over the entire specimen surface with an increase of about $20 \%$ near the specimen centers, and approaching $50 \%$ at the ends of the specimen. For both pressures used in the tests, the microhardness profiles were similar, indicating that the hardness of the specimens is a function of dislocation activity accommodating the imposed shear strain but not of the pressure under which the deformations were performed.

Microstructural observations were made using TEM and automated EBSD techniques. For the EBSD analysis, the samples were prepared by mounting the specimens in a transparent resin so that suitable cross-sections of the foils could be prepared. The samples were cut approximately along the slip direction with the cross section of sample exposed for characterization. The exposed surface was prepared using standard metallographic procedures. The regions scanned were approximately $0.15 \mathrm{~mm}$ near the specimen centers covering the entire thickness. Color coding of the orientations is indicated by the orientation color key shown with poles normal to the foil surface being represented (horizontal on the images shown). 
Figure 9 contains orientation images of the foil cross sections for the original foil (a), and for a specimen loaded to $4.2 \mathrm{GPa}$ and unloaded (b). Also shown are images of the specimen center region for a specimen deformed to a shear strain of 3.2.
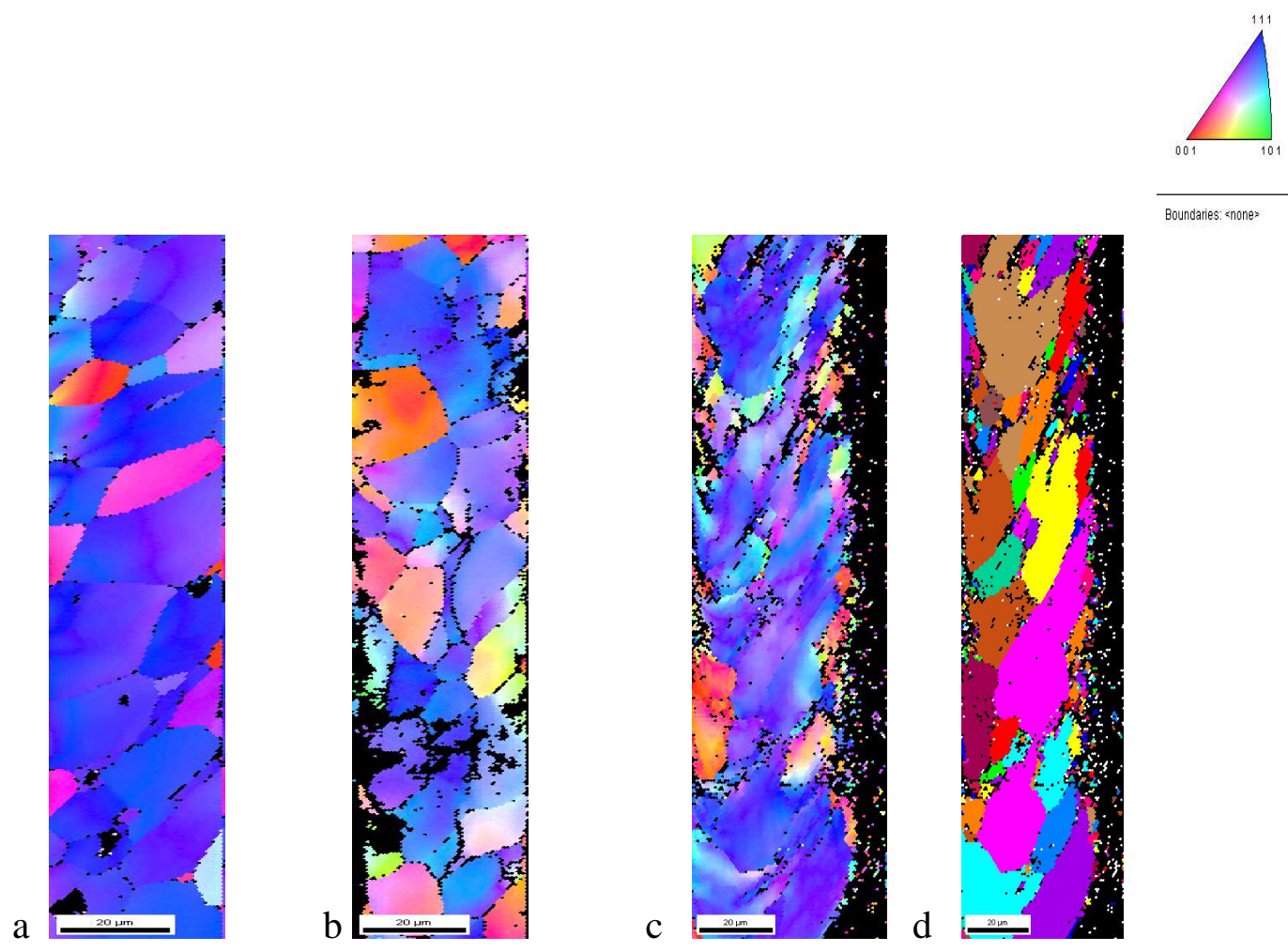

Boundarilis: : ₹none:

Figure 9 - OIM analysis of the cross section showing the grain behavior of the specimen subjected to 4.2 GPa a) Original specimen, $b$ ) held under HP $c, d)$ center sheared.

The Figure 9.a shows the inverse pole figure (IPF) results of the original specimen, the grains are well shaped and defined, a 111 texture is evident. Figure 9.b shows the lack of significant deformation for the specimen subjected to hydrostatic pressure. This result is consistent with the values of hardness obtained for 2.1 and $4.2 \mathrm{GPa}$, where there is no 
significant change in the microhardness and thus no change in the microstructure of the sample due to activation and interaction of dislocations.

Figure 9.c shows the center of sheared specimen, the grains look sheared and elongated along the slip direction, a 111 texture is also evident. In the edge of the sample a decrease in the image quality can be noticed, this is due to a higher dislocation activity taking place causing a multi-scattering of the diffracting electrons and thus lost of the EBSD image contrast in that region. A unique grain color map was also created for the sheared specimen. In this type of map color is assigned randomly to each of the grains in order to differentiate them from each other. From this map an approximate amount of strain can be indirectly extracted. In the case of the tests done at $4.2 \mathrm{GPa}$, a nominal strain of about 3.2 was read by the extensometers. Measuring the strain from the orientation image, using the observed shearing direction (indicated by a solid white line in Figure 9d) yields a strain of 3.0. This result gives assurance that the measurements taken by the extensometers, using the assumption that the specimen surfaces are "welded" to the deformation anvils, represent the actual strain experienced by the specimen.

To study the dislocation activity in detail both deformed and undeformed specimens were analyzed by bright field imaging in the TEM. Reported here are the results for specimens loaded to a pressure of $4.2 \mathrm{GPa}$.
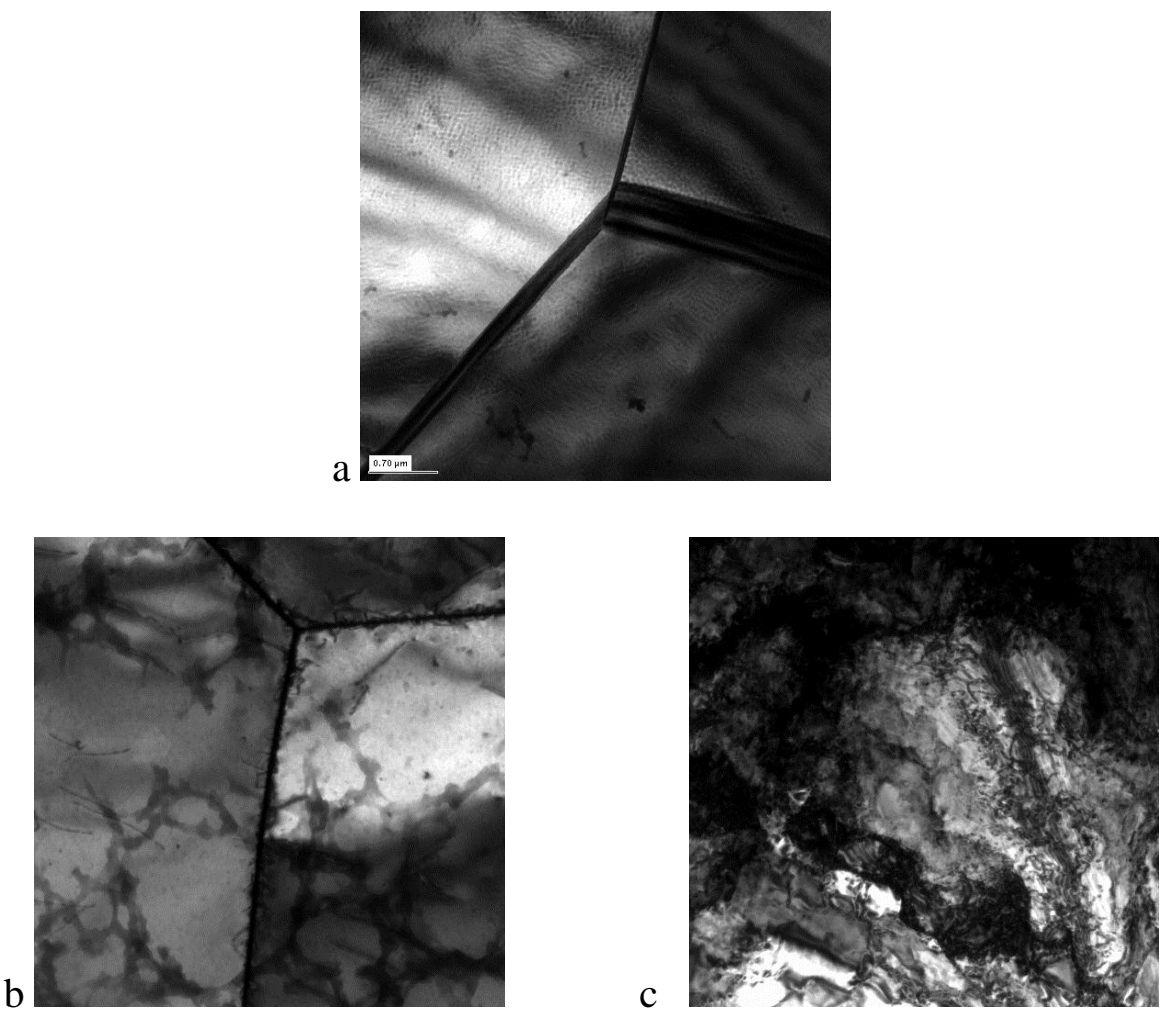

Figure 10 - TEM analysis of the samples at $4.2 \mathrm{GPa}$. a) Original specimen, b) specimen loaded and unloaded with no shearing, and c) specimen loaded and sheared. 
Figure 10.a shows the BF image of the original Ta foil that was used for the tests. No appreciable dislocation density prior to the deformation process is appreciable. For the specimen loaded to a pressure of $4.2 \mathrm{GPa}$ without shearing, a slight increase in dislocation content is observed, but the grains are still relatively free from dislocation debris. This explains why the measurements of the hardness in the specimens loaded and unloaded without shearing were close to those obtained for the undeformed specimens. It also helps to interpret the orientation images where some slight in-grain lattice distortion is observed due to the geometrically necessary component of the dislocation structure. These results show that while some dislocation motion occurs near the specimen centers during loading of the specimens, the crystallites remain largely undeformed. The structure therefore must undergo primarily elastic strain during loading to high pressures.

For the specimens loaded to high pressure followed by shearing, the TEM images show that the specimen contains a well-developed dislocation network. The TEM images of the specimens of both types of deformation are consistent with the observations obtained using hardness and EBSD techniques.

\section{Discussion of strain-stress results}

The finite element simulation and the experimental characterization of the samples held under hydrostatic pressure, established that the procedure described herein represents a reliable method to impose hydrostatic pressure on the thin foil specimens. This assertion was validated indirectly by applying characterization methods and measuring properties such as hardness, dislocation density and microstructure for the specimens subjected to such pressures. All properties measured after loading and unloading (with no shear deformation) were close to those from the original foils. After assuring in good confidence that the pressure attained was near the hydrostatic condition, specimens were deformed in shear to analyze their mechanical properties.

\section{Deformation of Polycrystalline Ta}

As stated previously, the samples were prepared with dimensions of about $3 \mathrm{~mm}$ in diameter and a nominal thickness of $50 \mu \mathrm{m}$. With these values and having three samples for a single test the load necessary to achieve the pressure of $1 \mathrm{GPa}$ was roughly about $22.3 \mathrm{kN}$ (5 kips). The samples were tested to roughly $50 \%$ of the capacity of the testing system which converts to a pressure of $4.2 \mathrm{GPa}$ for the given sample size. The stressstrain behavior is recorded Figure 11.

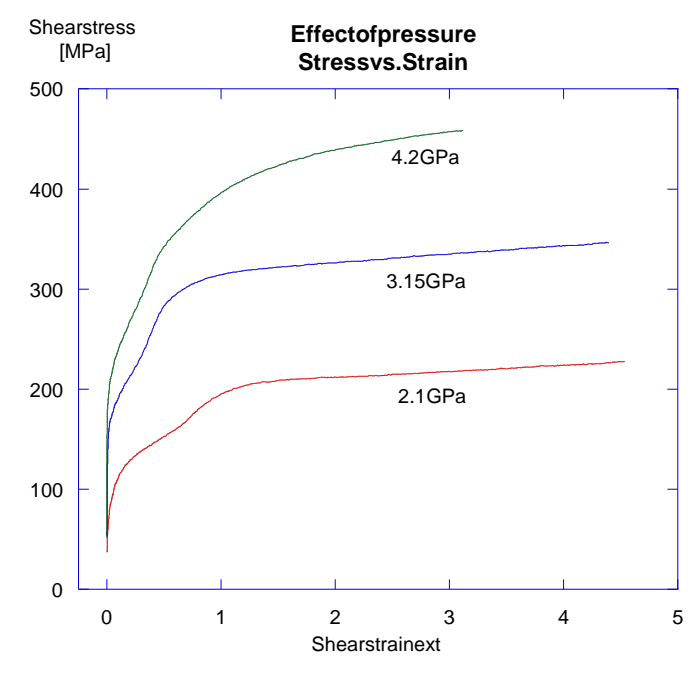


Figure 11 - Results of the effect of pressure on the stress vs. strain behavior of polycrystalline Ta foil.

The same general behavior for the stress-strain was exhibited for all pressures investigated. Three different stages are present during the deformation. The beginning of the test is the elastic region, this region exists to strains less than $2 \%$. After initial hardening, an intermediate zone is observed with a significantly reduced hardening rate, and a pressure-dependent inflection point. Finally, above a strain of 0.5 the material initiates a plateau zone (similar to stage IV hardening), which appears to be a steady state work hardening region observed [10] in the material.

By analysis of the small strain regions of the stress-strain curve, an estimate of the yield points can be obtained. This small strain region for the three pressures investigated is shown in Figure 12. It is immediately apparent that the yielding behavior is a strong function of the imposed pressure.

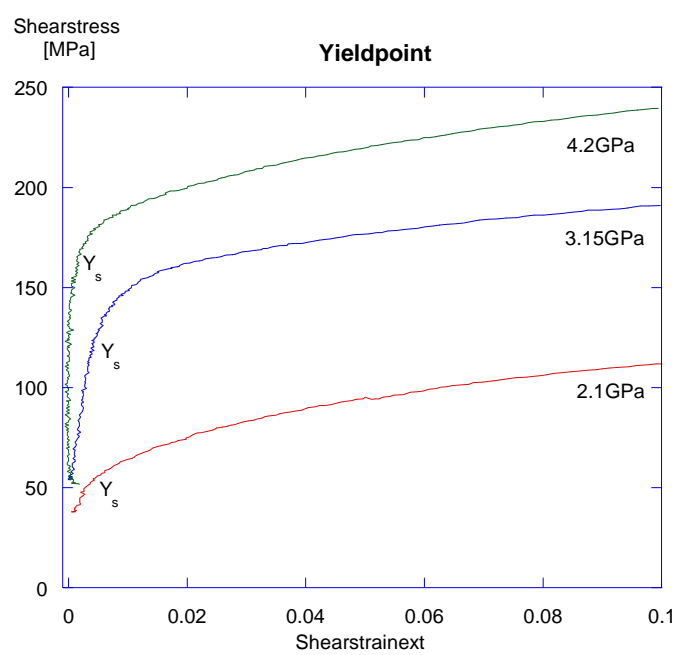

Figure 12 - Results of the effect of pressure on the Stress vs. strain behavior of poly Ta.

There are different approaches to study how dislocation dynamics is affected by hydrostatic pressure. Hydrostatic pressure constricts the dislocation movement, giving the materials a pressure-induced hardening effect, making it more difficult to deform the specimens with increasing pressure. This phenomenon can be observed easily in the figure 12. Some authors have reported the removal of the yielding point by applying hydrostatic pressure [18].

An approach to study how the pressure changes the mechanical behavior of materials was given by Steinberg and Guinan [14,15]. In their model the yield strength is found to be proportional to the shear modulus in the form 


$$
\left.\left.\frac{1}{Y_{0}} \frac{d Y}{d P}\right)_{0} \approx \frac{1}{G_{0}} \frac{d G}{d P}\right)_{0}
$$

So as the shear modulus increases with pressure so does the yield stress. The constitutive equations for both the shear modulus and yield strength are found to be

$$
G=G_{0}\left[1+\left(\frac{G_{P}^{\prime}}{G_{0}}\right) \frac{P}{\eta^{1 / 3}}\right]
$$

and

$$
Y=Y_{0} *\left[1+\beta\left(\varepsilon_{i}+\varepsilon\right)\right]^{n} *\left[1+\left(\frac{Y_{P}^{\prime}}{Y_{0}}\right) \frac{P}{\eta^{1 / 3}}\right]
$$

Where $\eta$ is the compressibility of the material, $P$ is the pressure, $\mathrm{G}$ the shear modulus, $Y$ is yield strength, $\beta$ and $n$ are work hardening parameters and $\varepsilon$ is the strain. Because the

\begin{tabular}{|c|c|}
\hline \multicolumn{2}{|c|}{ Table 1 } \\
\hline Pressure [GPa] & $\mathrm{Y}_{\mathrm{S}}[\mathrm{Mpa}]$ \\
\hline 2.1 & 54 \\
\hline 3.15 & 128 \\
\hline 4.2 & 165 \\
\hline
\end{tabular}
experiment was conducted at room temperature, the terms corresponding to temperature dependence can be neglected.

From these equations a close linear relation between the yield strength and the applied pressure is expected. In addition, if the values of the parameters for polycrystalline Tantalum [14] are used in the constitutive equation (Eq. 2), the expected yield strength under different pressures can be calculated and compared with the values obtained experimentally and shown in Table 1. This comparison is depicted in figure yield stress

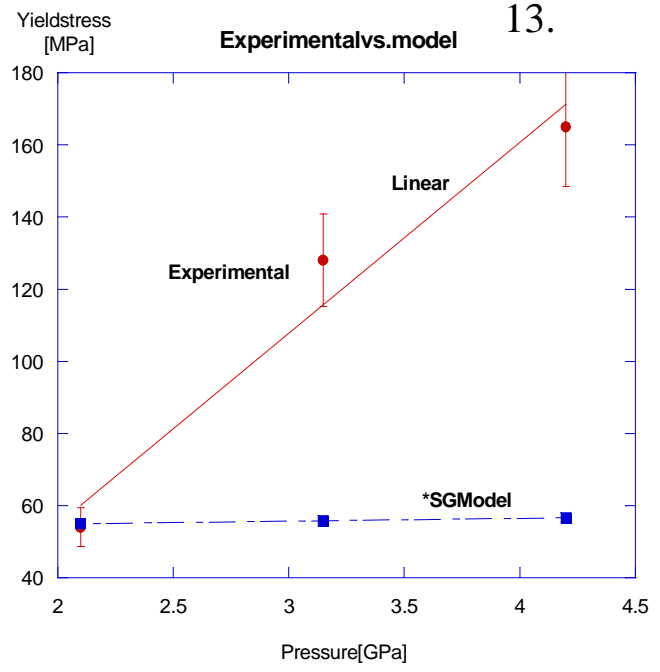

Figure 13 - Results of the effect of pressure on the stress vs. strain behavior of poly Ta.

A deviation from the model is evident with two maindiscrepancies between the expected and the experimental values. These discrepancies are in both the value and the behavior of the yield stress as a function of pressure. Two different fits were done for the experimental data, it is clear that the polynomial is more accurate than the linear predicted by the model for moderated pressures, this could be due to the fact that the experiment was done just after the limit in pressure studied by Steinberg-Guinan $(\leq$ 
2GPa). More evident deviation is that the model in the case of Ta predicts a small linear increase of about $3 \%$ in the yield strength but a more substantial is found experimentally, being of the order of 100 and $220 \%$ with pressure moderated increased up to $4.2 \mathrm{GPa}$

The measured yield strengths presented here, while much higher than those predicted by the Steinburg-Guinan model, lie significantly below the maximum values for yield in Ta established by their investigations,

$$
Y=Y_{0} *\left[1+\beta\left(\varepsilon_{i}+\varepsilon\right)\right]^{n} \leq Y_{\text {max }}
$$

where $Y_{\max }$ has been established to be $1.1 \mathrm{GPa}$ [14] in the case of Ta.

\section{Summary and Conclusion:}

A new procedure to study the mechanical properties of materials deformed by shearing strains while maintained under high pressure has been described. The paper focuses on the details of the experiment and qualitatively in the changes experienced by the specimens subjected to high pressures.

In order to use this procedure to study the properties of the mainly as a result of shear stresses, materials such as the dislocation mobility of single crystals, is desirable that the hydrostatic pressure contributes, in no way in the ideal case, or at least not in a significant level before the shear process. Based on the results exposed here, this procedure has proven to be a good method to study these properties. Although the optical microscopy analysis showed that the specimens maintained the deformed shape after unloading. The microstructure seems not to change due solely to the effect of the pressure applied. This was corroborated via Vickers characterization, with the hardness being almost equal to the un-deformed material. This was also validated through the EBSD and TEM imaging, with neither technique showing significant deformation of the microstructure nor dislocation multiplication.

Also the experiment allows the validation of models for materials held under high pressure. The Steinberg-Guinan model was tested using polycrystalline Ta. The outcome of these tests showed that hydrostatic pressure plays a more influent role than expected on properties such as yield strength, showing that it increases more rapidly than that predicted byavailable models.

\section{References:}

1. Paul McMillan "Chemistry of materials under extreme high pressure- high temperature conditions" Royal society of chemistry, 2003.

2. P. W. Bridgman, J. Appl. Phys. 24, 560, 1953.

3. S.R. Weir, J. Akella, C. Ruddle, T Goodwin and L. Siung. "Static strength of Ta and U under ultrahigh pressures.” Phys. Rev. B 11258-11265, 1998.

4. L.M. Hsiung and D.H. Lassila, "Shock-induced deformation twinning and omega transformation in tantalum and tantalum-tungsten alloys," Acta Mater. 48:4851-4865, 2000. 
5. P.Söderlind and J.A. Moriarty, "First-principles theory of Ta up to $10 \mathrm{Mbar}$ pressure: Structural and mechanical properties," Phys. Rev. B 57:10340-10350, 1998.

6. Lassila, "Strength of materials under high pressure", Report LLNL.

7. S. Duesbery and V. Vitek, "Plastic anisotropy in bcc transition metals," Acta Mater. 46:1481-1492, 1998.

8. L.H. Yang, P. Söderlind, J.A. Moriarty, "Atomistic simulation of pressure-dependent screw dislocations in bcc Ta," Mat. Sci. Eng. A309-310:102-107, 2001.

9. Elemental theory of crystal plasticity.

10. Dieter

11. PW Bridgman, "Effects of shearing stresses combined with high hydrostatic pressure", Phys. Rev. 48: 825-847 (1935)

12. PW Bridgman, "Effects of hydrostatic pressure on the plastic properties of the metals", Rev. Mod. Phys 17: 3-14 (1945)

13. PW Bridgman, "Shearing phenomena at high pressure", Phys. Rev. 48: 825-847 (1935)

14. DJ Steinberg, SG Cochran, MW Guinan "Constitutive model for metals applicable at high strain rate", J. Appl. Phys. 51: 1498- 1505 (1980).

15. D. Steinberg, D Breithaupt, C Honodel "Work-hardening and effective viscosity of solid beryllium", Physica 139 \& 140B: 762-765 (1986).

16. J.L. Lewandowski and P. Lowhaphandu, "Effect of hydrostatic pressure on mechanical behaviour and deformation processing of materials on materials", Int. Mat. Reviews, v 43, n 4, 1998, p 145-187

17. Seeger A. Mater Sci Eng A 2001;319-321:254.

18. Ito K, Vitek V. Phil Mag A 2001;81:1387.

Gumbsch P, Taeri-Baghbadrani S, Brunner D, Sigle W, Ru“hle M. Phys Rev Lett 2001;87:085505.

20. Jan Fikar1, Bernard Viguier2, Tomas Krumlı and Corinne Dupas, J. Phys.: Condens. Matter 14 (2002) 12887-12895. 